\title{
Integrated Bioinformatics analysis and clinical validation reveals that high expression of mucin 1 in intrahepatic cholangiocarcinoma predicts recurrence after curative resection
}

\author{
FEI-YU CHEN $^{1 *}$, CHENG ZHOU $^{1 *}$, XIANG-YU ZHANG ${ }^{1 *}$, KAI-QIAN ZHOU $^{1}$, \\ YUAN-FEI PENG ${ }^{1}$, LEI YU ${ }^{1}$, JIA FAN ${ }^{1-4}$, JIAN ZHOU $^{1-4}$, JIE HU $^{1}$ and ZHENG WANG ${ }^{1}$ \\ ${ }^{1}$ Department of Liver Surgery, Liver Cancer Institute, Zhongshan Hospital, Fudan University, \\ Key Laboratory of Carcinogenesis and Cancer Invasion, Fudan University, Ministry of Education; \\ ${ }^{2}$ State Key Laboratory of Genetic Engineering and Collaborative Innovation Center for Genetics and Development, \\ School of Life Sciences, Fudan University; ${ }^{3}$ Shanghai Key Laboratory of Organ Transplantation, Zhongshan Hospital, \\ Fudan University; ${ }^{4}$ Institute of Biomedical Sciences, Fudan University, Shanghai 200032, P.R. China
}

Received December 13, 2018; Accepted June 13, 2019

DOI: 10.3892/etm.2020.9178

\begin{abstract}
Intrahepatic cholangiocarcinoma (ICC) is a cancer type with high malignancy and a current lack of biomarkers to predict recurrence. In the present study, to identify potential biomarkers, five ICC datasets from the Gene Expression Omnibus database were analyzed to construct initial datasets by using a robust rank aggregation approach. A total of 19 upregulated genes were identified in the initial datasets. The genes identified were then further analysed using data from The Cancer Genome Atlas. Only mucin 1 (MUC1) exhibited significance regarding differential expression and survival prediction. Finally, the expression levels of MUC1 were assessed using reverse transcription-quantitative PCR in 61 pairs of ICC tumor and matched non-cancerous samples. The expression of MUC1 was significantly elevated in ICC tissues compared with that in matched non-cancerous counterparts $(\mathrm{P}=0.001)$. Patients with high MUC1 expression levels had
\end{abstract}

Correspondence to: Professor Zheng Wang or Dr Jie Hu, Department of Liver Surgery, Liver Cancer Institute, Zhongshan Hospital, Fudan University, Key Laboratory of Carcinogenesis and Cancer Invasion, Fudan University, Ministry of Education, 180 Fenglin Road, Shanghai 200032, P.R. China

E-mail:wzdoc@163.com

E-mail: hujies202@163.com

${ }^{*}$ Contributed equally

Abbreviations: adjP, Bonferroni correction adjusted P-value; GEO, Gene Expression Omnibus; ICC, intrahepatic cholangiocarcinoma; $\log \mathrm{FC}, \log 2$-transformed fold change; MUC1, mucin 1; OS, overall survival; RRA, robust rank aggregation; RFS, recurrence-free survival; TCGA, The Cancer Genome Atlas

Key words: intrahepatic cholangiocarcinoma, Bioinformatics analysis, mucin 1 , gene dysregulation significantly shorter overall survival $(\mathrm{OS}, \mathrm{P}=0.009)$ and recurrence-free survival (RFS, $\mathrm{P}=0.012$ ). MUC1 was identified as an independent prognostic factor for OS [hazard ratio $(\mathrm{HR})=2.364,95 \% \mathrm{CI}: 1.214-4.485 ; \mathrm{P}=0.023$ ] and $\mathrm{RFS}$ $(\mathrm{HR}=2.552$ 95\%CI: 1.294-5.032; $\mathrm{P}=0.007)$ in the multivariate analysis. Using receiver operating characteristic analysis, a co-index including MUC1 had a high accuracy for predicting survival [MUC1 combined with serum levels of CEA and cancer antigen 19-9, and lymph node metastasis, area under curve $(\mathrm{AUC})=0.746,95 \% \mathrm{CI}$ : 0.620-0.872] and recurrence (MUC1 combined with bile duct invasion and lymph node metastasis, $\mathrm{AUC}=0.729,95 \% \mathrm{CI}$ : 0.605-854). In conclusion, MUC1 is highly expressed in ICC tissue and is a potential prognostic biomarker and therapeutic target for ICC.

\section{Introduction}

Intrahepatic cholangiocarcinoma (ICC) is the second most common type of primary liver malignancy after hepatocellular carcinoma $(1,2)$. ICC originates from the intrahepatic bile duct and affected patients present with a focal liver mass (3). The incidence of ICC is increasing worldwide and mortality rates have not declined (4-6). The poor prognosis of ICC is of particular concern. Resection is the only curative treatment for ICC, but even with resection, patients with ICC have a poor prognosis $(7,8)$. For early ICC, the 5-year survival of patients with resection is around $30-35 \%$ (9).

Gene dysregulation contributes to the tumorigenesis of ICC (10). Identification of the dysregulated genes may be used to identify novel treatment targets and predictors for response to chemotherapy and radiotherapy (11). Numerous genes appear to be promising diagnostic biomarkers for ICC, as well as predictors of prognosis and targets for therapy $(10,12,13)$. Gene expression studies are being used to identify these genes.

Integrated data analysis has a key role in the analysis of high-throughput data (14). The R package RobustRankAggreg may be used for data integration with the robust rank aggregation (RRA) method (15). The algorithm assigns a P-value 
to each item that indicates how much better it is positioned in the ranked list than expected by chance. This P-value is used for re-ranking the items and to determine statistical significance (16).

In the present study, an integrated Bioinformatics analysis was used to identify genes associated with ICC.Clinical samples were then analyzed to validate a gene that was identified and its clinical significance.

\section{Patients and methods}

Data selection and datasets. A search for ICC expression profiling array data was performed using Gene Expression Omnibus (GEO; www.ncbi.nlm.nih.gov/geo/) (17) for entries added until May 2017. The search strategy was based on a combination of the following terms: \{(intrahepatic cholangiocarcinoma) OR [cholangio* AND (cancer* OR tumor* OR tumor* OR carcinoma)]\}. All data series were carefully screened. Studies on more than one type of tumor, but that included ICC, were also evaluated. Studies that profiled different histologic subtypes but that did not include non-cancerous tissue, and studies using only cell lines, were excluded. Gene expression data and corresponding clinical information for the cholangiocarcinoma dataset were downloaded from The Cancer Genome Atlas (TCGA, https://www. cancer.gov/tcga) data portal in May 2017.

Dataset construction. The series of data from the GEO datasets (GSE89749, GSE76297, GSE57555, GSE32879 and GSE26566) were analyzed separately using the limma application (version 3.30.13) (18). The expression level of each gene was $\log 2$-transformed for further analysis. Bonferroni correction was used to adjust P-values; a correction that adjusted each $\mathrm{P}$-value $(\operatorname{adjP})<0.01$ for a gene with a lfold change $(\mathrm{FC}) \mid>2$ was applied to each data series. Identification of dysregulated genes associated with cholangiocarcinoma was performed using the edgeR application (version 3.16.5) (19). $\log 2$-transformation and Bonferroni correction were also applied for each gene. For a more stringent limit, the applied adjusted $\mathrm{P}$-value was $<0.001$ and the llog2-transformed $\mathrm{FCl}$ was $>4$.

RRA was used for the integration of the GEO data series. The R package (version: 1.1) was used to detect genes that were ranked consistently better than expected under the null hypothesis of uncorrelated inputs and to assign a significance score for each gene (16). All genes identified were statistically significant $(\mathrm{P}<0.01)$ after the Bonferroni correction was performed.

Validation of potential dysregulated genes using reverse transcription-quantitative PCR (RT-qPCR). A total of 61 pairs of ICC and adjacent non-cancerous tissues were used to validate the potential dysregulated genes. All tissues were ground separately in liquid nitrogen. The total RNA was extracted using TRIzol reagent (Invitrogen; Thermo Fisher Scientific, Inc.). Complementary DNA synthesis was performed using a PrimeScript ${ }^{\mathrm{TM}}$ reverse transcriptase reagent kit (Takara Bio, Inc.). Amplification and quantification were performed using the ABI PRISM 7900 Sequence Detection System (Applied Biosystems; Thermo Fisher Scientific, Inc.) and SYBR ${ }^{\circledR}$ Premix Ex Taq ${ }^{\mathrm{TM}}$ (Tli RNaseH Plus; Takara Bio, Inc.).
GAPDH was used as an endogenous control. The expression level of the analyte gene was normalized to that of GAPDH and the $2^{-\Delta \Delta \mathrm{Cq}}$ method was applied (20). The primers used are presented in Table SI.

Patients. The study protocol was approved by the Clinical Research Ethics Committee of Zhongshan Hospital, Fudan University (Shanghai, China). ICC was defined as adenocarcinoma arising from the second-order or greater distal branches of the intrahepatic bile ducts. The inclusion criteria were as follows: i) The patient received a curative resection between January 2009 and December 2012 at Zhongshan Hospital, Fudan University (Shanghai, China); ii) the patient's ICC was diagnosed by two experienced pathologists; iii) each patient received the operation from the same surgical group; iv) no concurrent malignant tumors of other types were present; v) no anti-tumor treatments were given prior to the surgery; vi) the complete follow-up information was available. All tissues were frozen immediately after the hepatectomies.

The tumor-nodes-metastasis staging was performed according to the guidelines of the eighth edition of the American Joint Committee on Cancer/International Union against Cancer (21).

Follow-up. After resection, each patient had an appointment for a follow-up exam every 3-4 months during the first 2 years, and then every 4-6 months during the next year. Liver function indicators, serum $\alpha$-fetoprotein and hematological parameters were examined at each follow-up visit. Liver ultrasonography was performed at each visit by physicians who were not involved in the patient's treatment and had no access to treatment information. Contrast computed tomography (CT) scans of the chest, abdomen and pelvis were performed once every 6 months. A bone scan or a magnetic resonance imaging (MRI) scan was performed if it was considered necessary due to any clinical indication. If tumor recurrence in the liver was suspected, a CT scan or MRI with intravenous contrast was performed. Biopsies of lesions were performed when a definite diagnosis could not be elucidated made by other methods.

Statistical analysis. All R packages used were based on $\mathrm{R}$ software (version 3.3.2). Comparison of expression levels between tumors and paired adjacent non-cancerous tissues was performed using paired t-tests.

Overall survival (OS) time was measured from the date of surgery to the date of death. The recurrence-free survival (RFS) time was calculated from the date of surgery to the date of the first clinically-documented tumor recurrence or metastasis, or the date of death. Inter-group comparisons were performed using the chi-squared or Fisher's exact test. The OS and RFS times were calculated using the Kaplan-Meier method; log-rank tests were used to assess differences between survival curves. The Cox linear hazards model was used logistic regression analysis for the univariate and multivariate analysis. The accuracy of predicting prognosis was evaluated using receiver operating characteristic (ROC) curves, and the predictive performance was determined by calculating the area under the ROC curve (AUC). All statistical analyses were performed using the SPSS 22.0 software 
package (IBM Corp.). $\mathrm{P}<0.05$ was considered to indicate statistical significance.

\section{Results}

Characteristics of the data series and associated dysregulated genes. A total of five data series from GEO datasets deposited between 2012 and 2017 were included for further analysis (22-26). The characteristics of these studies/data series are presented in Table I.

A total of 345 tumor samples and 175 control counterparts were included in the integrated dataset. The control samples varied across the studies (Table I). Different microarray platforms were used in the studies; the number of mRNA probes ranged from 22,185 to 70,753 . Use of the limma $\mathrm{R}$ package $(\log \mathrm{FC}>2$, adj $\mathrm{P}<0.01)$ revealed that at least 31 dysregulated genes were contained in one data series. In total, the median number of significantly upregulated genes was 46 (range, 14-4,889), and the median number of downregulated genes was 534 (range, 17-3,801) (Table I).

The RRA analysis identified 19 upregulated genes and 130 downregulated genes (Table SII). The P-values for all dysregulated genes were statistically significant $(\operatorname{adj} \mathrm{P}<0.01)$ after Bonferroni correction. The corrected P-values for the dysregulated genes ranged from $1.38 \times 10^{-7}$ to $9.86 \times 10^{-3}$. To identify specific biomarkers, the 19 upregulated genes were selected for further analysis (Fig. 1A and Table II).

Mucin 1 (MUC1) is upregulated in ICC and has clinical significance in TCGA data. Data from the TCGA were used to study the upregulated genes identified. There were 36 ICC tumor tissues and nine normal tissues in the TCGA dataset. The edgeR package was used to reveal the dysregulated genes. A total of 769 upregulated genes and 713 downregulated genes were identified $(\log \mathrm{FC}>4, \operatorname{adj} \mathrm{P}<0.001)$. The results for the upregulated genes are presented in Fig. 1B and Table SIII. Intersection of the two datasets revealed that 13 upregulated genes were identified in the GEO as well as TCGA datasets (Fig. 1C). All 13 genes were upregulated in ICC tissue compared with normal liver tissue (Fig. 1D and E), but only MUC1 had a statistically significant impact in the survival analysis (Figs. 1F and S1). The expression of MUC1 in ICC patients was selected for further study.

Validation of MUC1 expression in patients with ICC. A total of 61 patients with ICC met the inclusion criteria and were enrolled in the present study. All of them had ICC that was diagnosed and pathologically confirmed at the Liver Surgery Department of Zhongshan Hospital, Fudan University (Shanghai, China) between January 2009 and December 2012. The clinicopathological characteristics of these patients are presented in Table III.

MUC1 expression was examined using RT-qPCR analysis. Consistent with the results of the dataset analysis, the expression of MUC1 was significantly higher in ICC tumor tissue than in adjacent non-tumor tissue ( $\mathrm{P}=0.001$; Fig. $2 \mathrm{~A})$. The enrolled patients were divided into two groups based on the median MUC1 expression value (i.e., low MUC1 expressed in ICC tissue, MUC1 $1^{\text {low }}, \mathrm{n}=30$; high MUC1 expressed in ICC tissue, $\mathrm{MUCl}^{\text {high }}, \mathrm{n}=31$ ). Inter-group comparisons of the

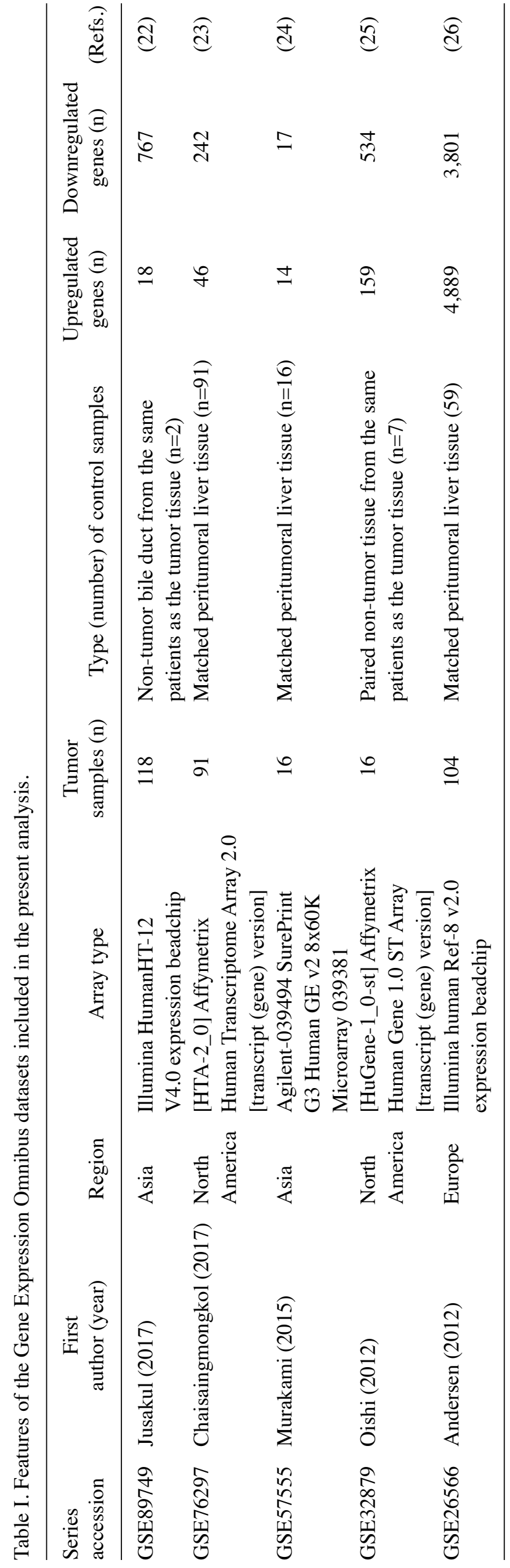




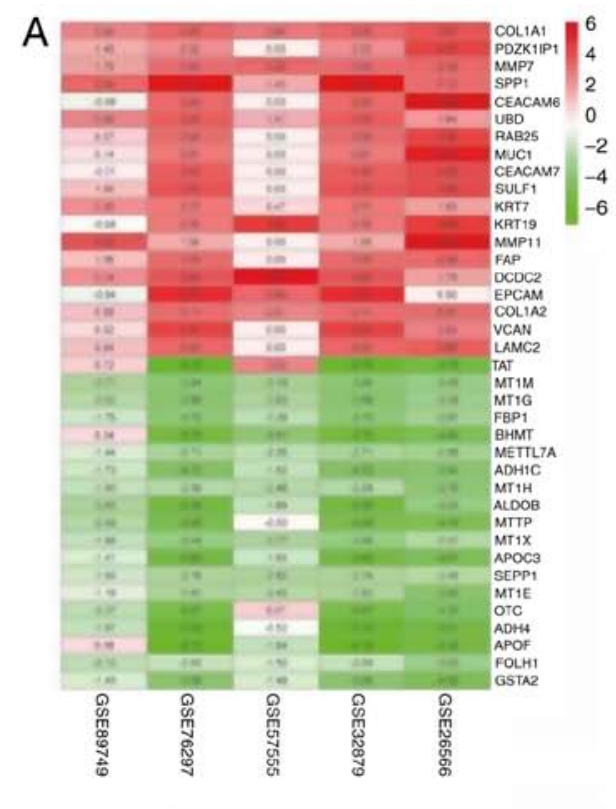

B
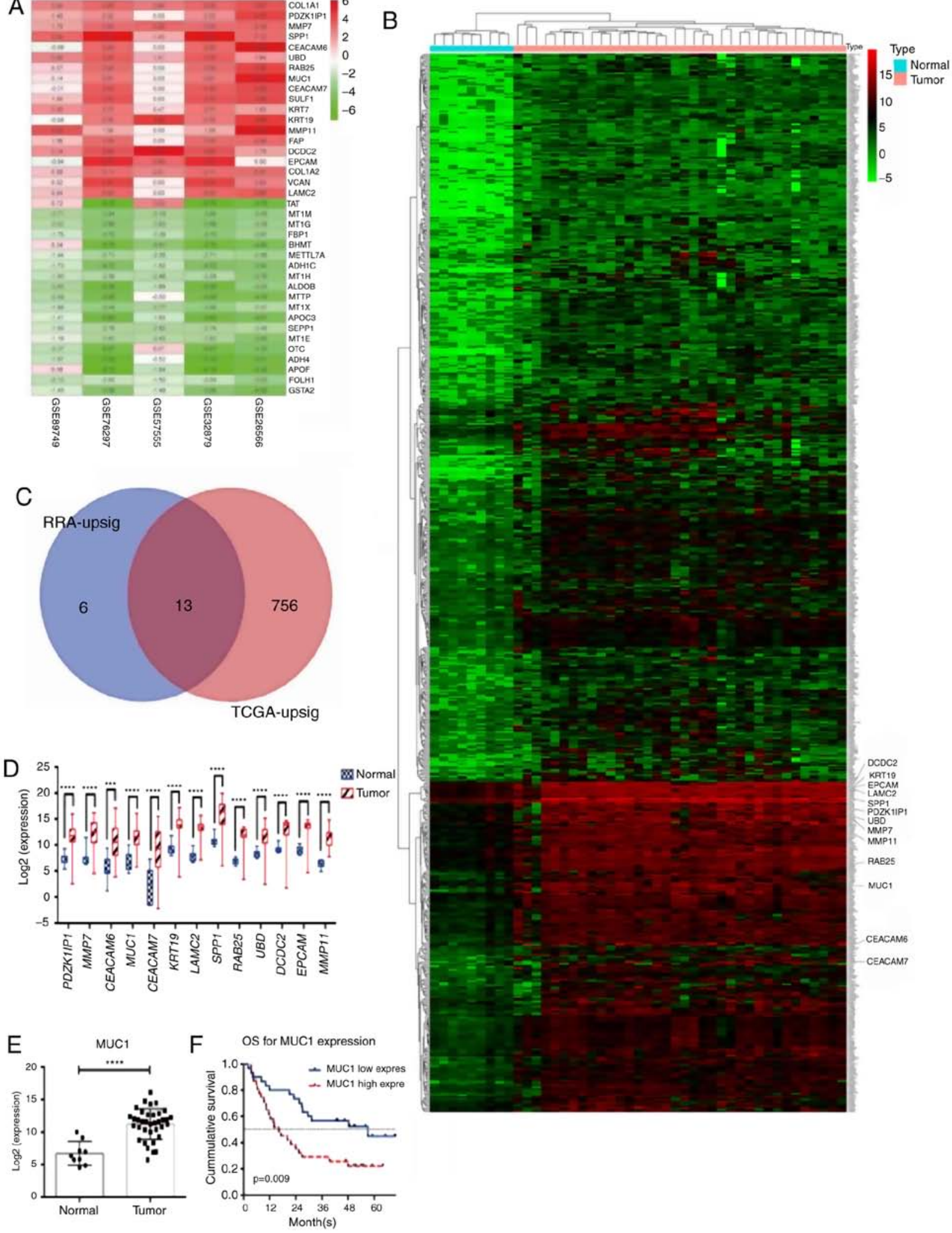

Figure 1. MUC1 is upregulated in ICC tissue, as validated using databases. (A) The 19 upregulated genes and the 19 most significant downregulated genes linked to cholangiocarcinoma identified using robust rank aggregation. Numbers in the colored fields indicate the log2-transformed fold change. 0.00 or white boxes indicates that the gene was absent from the respective dataset. (B) Upregulated genes linked to cholangiocarcinoma identified using the edgeR package (TCGA dataset). The enlarged gene symbols adjacent to the heatmap are the 13 intersected genes. (C) 13 genes were identified to be upregulated in the RRA integrated datasets and the TCGA data. (D and E) The expression levels of (D) the 13 genes and (E) MUC1 were significantly higher in ICC tissue, validated using TCGA data. (F) Among the 13 genes, only MUC1 predicted OS. RRA-upsig refers to the significantly upregulated genes identified using RRA; TCGA-upsig refers to significantly upregulated genes among the TCGA data. ${ }^{* * *} \mathrm{P}<0.001$; ${ }^{* * * *} \mathrm{P}<0.0001$. MUC1, mucin 1 ; TCGA, The Cancer Genome Atlas; ICC, intrahepatic cholangiocarcinoma; OS, overall survival; RRA, robust rank aggregation; PDZK1IP1, PDZK1 Interacting Protein 1; MMP7, matrix metalloproteinase 7; CEACAM6, carcinoembryonic antigen related adhesion molecules 6; MUC1, mucin 1; CEACAM7, carcinoembryonic antigen related adhesion molecules 7; KRT19, keratin 19; LAMC2, laminin subunit gamma-2; SPP1, secreted phosphoprotein 1; RAB25, Ras Genes from Brain Protein 25; UBD, ubiquitin D; DCDC2, doublecortin domain containing 2; EPCAM, epithelial cell adhesion molecule; MMP11, matrix metalloproteinase 11. 
Table II. The 19 upregulated genes and the 19 most significant downregulated genes associated with cholangiocarcinoma, identified using robust rank aggregation.

\begin{tabular}{|c|c|c|c|}
\hline Gene symbol & P-value & Adjusted P-value & $\log \mathrm{FC}$ \\
\hline COL1A1 & $4.14 \times 10^{-11}$ & $2.22 \times 10^{-6}$ & 3.147 \\
\hline PDZK1IP1 & $1.08 \times 10^{-9}$ & $5.82 \times 10^{-5}$ & 2.093 \\
\hline MMP7 & $2.16 \times 10^{-9}$ & $1.16 \times 10^{-4}$ & 2.864 \\
\hline SPP1 & $2.99 \times 10^{-9}$ & $1.61 \times 10^{-4}$ & 3.904 \\
\hline CEACAM6 & $5.02 \times 10^{-9}$ & $2.70 \times 10^{-4}$ & 2.195 \\
\hline UBD & $1.26 \times 10^{-8}$ & $6.79 \times 10^{-4}$ & 2.610 \\
\hline RAB25 & $1.50 \times 10^{-8}$ & $8.06 \times 10^{-4}$ & 2.208 \\
\hline MUC1 & $2.06 \times 10^{-8}$ & $1.11 \times 10^{-3}$ & 2.284 \\
\hline CEACAM7 & $2.74 \times 10^{-8}$ & $1.47 \times 10^{-3}$ & 2.174 \\
\hline SULF1 & $3.78 \times 10^{-8}$ & $2.03 \times 10^{-3}$ & 2.511 \\
\hline KRT7 & $4.01 \times 10^{-8}$ & $2.16 \times 10^{-3}$ & 2.040 \\
\hline KRT19 & $4.26 \times 10^{-8}$ & $2.29 \times 10^{-3}$ & 2.819 \\
\hline MMP11 & $4.89 \times 10^{-8}$ & $2.63 \times 10^{-3}$ & 2.526 \\
\hline FAP & $5.34 \times 10^{-8}$ & $2.87 \times 10^{-3}$ & 2.099 \\
\hline DCDC2 & $5.95 \times 10^{-8}$ & $3.20 \times 10^{-3}$ & 3.545 \\
\hline EPCAM & $6.92 \times 10^{-8}$ & $3.72 \times 10^{-3}$ & 2.401 \\
\hline COL1A2 & $1.36 \times 10^{-7}$ & $7.34 \times 10^{-3}$ & 2.544 \\
\hline VCAN & $1.56 \times 10^{-7}$ & $8.37 \times 10^{-3}$ & 2.353 \\
\hline LAMC2 & $1.83 \times 10^{-7}$ & $9.86 \times 10^{-3}$ & 2.228 \\
\hline
\end{tabular}

B, Downregulated

\begin{tabular}{llcr}
\hline Gene symbol & P-value & Adjusted P-value & logFC \\
\hline TAT & $2.57 \times 10^{-12}$ & $1.38 \times 10^{-7}$ & -3.201 \\
MT1M & $1.18 \times 10^{-11}$ & $6.32 \times 10^{-7}$ & -3.387 \\
MT1G & $2.20 \times 10^{-11}$ & $1.18 \times 10^{-6}$ & -3.279 \\
FBP1 & $7.71 \times 10^{-11}$ & $4.15 \times 10^{-6}$ & -2.892 \\
BHMT & $8.03 \times 10^{-11}$ & $4.32 \times 10^{-6}$ & -3.980 \\
METTL7A & $8.36 \times 10^{-11}$ & $4.49 \times 10^{-6}$ & -2.438 \\
ADH1C & $8.58 \times 10^{-11}$ & $4.62 \times 10^{-6}$ & -3.404 \\
MT1H & $8.81 \times 10^{-11}$ & $4.74 \times 10^{-6}$ & -2.657 \\
ALDOB & $1.03 \times 10^{-10}$ & $5.54 \times 10^{-6}$ & -3.811 \\
MTTP & $1.42 \times 10^{-10}$ & $7.62 \times 10^{-6}$ & -3.601 \\
MT1X & $2.89 \times 10^{-10}$ & $1.56 \times 10^{-5}$ & -2.816 \\
APOC3 & $3.31 \times 10^{-10}$ & $1.78 \times 10^{-5}$ & -3.859 \\
SEPP1 & $4.31 \times 10^{-10}$ & $2.32 \times 10^{-5}$ & -2.490 \\
MT1E & $6.40 \times 10^{-10}$ & $3.44 \times 10^{-5}$ & -2.646 \\
OTC & $7.06 \times 10^{-10}$ & $3.79 \times 10^{-5}$ & -3.225 \\
ADH4 & $7.06 \times 10^{-10}$ & $3.80 \times 10^{-5}$ & -4.379
\end{tabular}

Table II. Continued.

B, Downregulated

\begin{tabular}{lccc}
\hline Gene symbol & P-value & Adjusted P-value & $\operatorname{logFC}$ \\
\hline APOF & $7.06 \times 10^{-10}$ & $3.80 \times 10^{-5}$ & -3.828 \\
FOLH1 & $7.43 \times 10^{-10}$ & $3.99 \times 10^{-5}$ & -2.194 \\
GSTA2 & $7.95 \times 10^{-10}$ & $4.28 \times 10^{-5}$ & -3.152 \\
\hline
\end{tabular}

P-values were adjusted using Bonferroni correction. LogFC, $\log 2$-transformed fold change. COL1A1, collagen type I alpha 1 chain; PDZK1IP1, PDZK1 Interacting Protein 1; MMP7, matrix metalloproteinase 7; SPP1, secreted phosphoprotein 1; CEACAM6, carcinoembryonic antigen related adhesion molecules 6; UBD, ubiquitin D; RAB25, Ras Genes from Brain Protein 25; MUC1, mucin 1; CEACAM7, carcinoembryonic antigen related adhesion molecules 7; SULF1, Sulfatase 1; KRT7, keratin 7; KRT19, keratin 19; MMP11, matrix metalloproteinase 11; FAP, Fibroblast Activation Protein Alpha; DCDC2, doublecortin domain containing 2; EPCAM, epithelial cell adhesion molecule; COL1A2, collagen type I alpha 2 chain; VCAN, versican; LAMC2, laminin subunit gamma-2. TAT, tyrosine aminotransferase; MT1M, metallothionein 1M; MT1G, metallothionein 1G; FBP1, fructose-bisphosphatase 1; BHMT, betaine-homocysteine S-methyltransferase; METTL7A, methyltransferase like 7A; ADH1C, alcohol dehydrogenase 1C (Class I), gamma polypeptide; MT1H, metallothionein $1 \mathrm{H}$; ALDOB, aldolase, fructose-bisphosphate B; MTTP, microsomal triglyceride transfer protein; MT1X, metallothionein 1X; APOC3, apolipoprotein $\mathrm{C} 3$; SEPP1, selenop; MT1E, metallothionein 1E; OTC, ornithine carbamoyltransferase; ADH4, alcohol dehydrogenase 4 (Class II), Pi Polypeptide; APOF, apolipoprotein F; FOLH1, folate hydrolase 1; GSTA2, glutathione S-transferase alpha 2.

clinicopathological characteristics are presented in Table III. The MUC1 ${ }^{\text {high }}$ group included more patients with tumors that invaded the surrounding tissue $(\mathrm{P}=0.040)$. Inter-group differences for the other clinicopathological characteristics were not statistically significant. The levels of MUC1 expression in tumors that invaded the surrounding tissue were higher than in those without surrounding tissue invasion ( $\mathrm{P}=0.027$; Fig. $2 \mathrm{~B})$.

Clinical validation of the prognostic significance of MUC1 expression. Survival analysis was performed to identify whether MUC1 levels may be used to predict prognosis in ICC patients. The median survival times of the $\mathrm{MUC1}^{\text {low }}$ and MUC $1^{\text {high }}$ groups were 55.06 and 17.25 months, respectively $(\mathrm{P}=0.009)$. The 1-, 3- and 5-year OS rates for the $\mathrm{MUC1}^{\text {low }}$ group were 73,57 and $45 \%$, respectively, as opposed to 35, 26 and $20 \%$, respectively, for the MUC1 ${ }^{\text {high }}$ group. The differences in OS rates according to the log-rank test was statistically significant between the two groups $(\mathrm{P}=0.009$; Fig. 2C). A total of 9 patients (30.0\%) in the MUC1 $1^{\text {low }}$ group and $17(54.8 \%)$ in the MUC1 $1^{\text {high }}$ group experienced tumor recurrence. The inter-group difference in the RFS rates was statistically significant ( $\mathrm{P}=0.012$; Fig. 2D).

The results of the univariate analysis indicated that MUC1 expression levels were a significant prognostic factor for OS [hazard ratio $(\mathrm{HR})=2.233$, 95\% CI: $1.214-4.485, \mathrm{P}=0.011$; Table IV]. The serum levels of carcinoembryonic antigen 
Table III. Clinical characteristics of enrolled patients.

\begin{tabular}{|c|c|c|c|c|}
\hline Parameter & All patients $(\mathrm{n}=61)$ & MUC1 $1^{\text {low }}(n=30)$ & $\mathrm{MUC}^{\text {high }}(\mathrm{n}=31)$ & P-value ${ }^{a}$ \\
\hline Age (years) & $59(39-77)$ & $57(39-72)$ & $59(40-77)$ & 0.86 \\
\hline Gender & & & & 0.21 \\
\hline Male & $40(65.57)$ & $22(73.33)$ & $18(58.06)$ & \\
\hline Female & $21(34.43)$ & $8(26.67)$ & $13(41.94)$ & \\
\hline Number of tumors & & & & 0.85 \\
\hline Single & $42(68.85)$ & $24(80.00)$ & $18(58.06)$ & \\
\hline Multiple & $19(31.15)$ & $6(20.00)$ & $13(41.94)$ & \\
\hline Tumor size $(\mathrm{cm})$ & $6(2-14)$ & $6(2-11.5)$ & $6(2.5-14)$ & 0.79 \\
\hline Vascular invasion & & & & 0.28 \\
\hline Yes & $16(26.23)$ & $6(20.00)$ & $10(32.26)$ & \\
\hline No & 45 (73.77) & $24(80.00)$ & $21(67.74)$ & \\
\hline Bile duct invasion & & & & 0.08 \\
\hline Yes & $6(9.84)$ & $5(16.67)$ & $1(3.23)$ & \\
\hline No & $55(90.16)$ & $25(83.33)$ & $30(96.77)$ & \\
\hline Nerve invasion & & & & 0.53 \\
\hline Yes & $3(4.92)$ & $2(6.67)$ & $1(3.23)$ & \\
\hline No & $58(95.08)$ & $28(93.33)$ & $30(96.77)$ & \\
\hline T stage & & & & 0.16 \\
\hline T1a & $14(22.95)$ & $9(30.00)$ & $5(16.13)$ & \\
\hline $\mathrm{T} 1 \mathrm{~b}$ & $17(27.87)$ & $8(26.67)$ & $9(29.03)$ & \\
\hline $\mathrm{T} 2$ & $26(42.62)$ & $13(43.33)$ & $13(41.94)$ & \\
\hline $\mathrm{T} 3$ & $0(0.00)$ & $0(0.00)$ & $0(0.00)$ & \\
\hline $\mathrm{T} 4$ & $4(6.56)$ & $0(0.00)$ & $4(12.90)$ & \\
\hline LN metastasis & & & & 0.53 \\
\hline Yes & $3(4.92)$ & $2(6.67)$ & $1(3.23)$ & \\
\hline No & $58(95.08)$ & $28(93.33)$ & $30(96.77)$ & \\
\hline Surrounding tissue invasion & & & & 0.04 \\
\hline Yes & $4(6.56)$ & $0(0.00)$ & $4(12.90)$ & \\
\hline No & $57(93.44)$ & $30(100.00)$ & $27(87.10)$ & \\
\hline Differentiation & & & & 0.11 \\
\hline Well & $50(81.97)$ & $27(90.00)$ & $23(74.19)$ & \\
\hline Poor & $11(18.03)$ & $3(10.00)$ & $8(25.81)$ & \\
\hline Type of resection & & & & 0.29 \\
\hline Less than hemihepatectomy & $46(75.41)$ & $21(70.00)$ & $25(80.65)$ & \\
\hline Hemihepatectomy & $13(21.31)$ & $7(23.33)$ & $6(19.35)$ & \\
\hline Extended hemihepatectomy & $2(3.28)$ & $2(6.67)$ & $0(0.00)$ & \\
\hline Post-operative treatment & & & & 0.311 \\
\hline Yes & $9(14.75)$ & $3(10.00)$ & $6(19.35)$ & \\
\hline No & $42(68.85)$ & $27(90.00)$ & $25(80.65)$ & \\
\hline
\end{tabular}

Values are expressed as the median (range) or $\mathrm{n}(\%)$. Vascular invasion, bile duct invasion and nerve invasion were identified pathologically. Post-operative treatment includes transarterial chemoembolization or radiofrequency ablation. LN, lymph node; MUC1, mucin 1. ${ }^{\text {aP-values }}$ represent comparison between $\mathrm{MUC} 1^{\text {low }}$ group and $\mathrm{MUC}^{\text {high }}$ group.

(CEA) and cancer antigen 19-9 (CA19-9), the degree of tumor differentiation, tumor diameter, tumor $\mathrm{T}$ stage and presence of lymph node metastasis were also significant influencing factors of OS (Table IV). The multivariate analysis revealed that a high MUC1 expression level $(\mathrm{HR}=2.364,95 \% \mathrm{CI}$ : 1.127-4.960, $\mathrm{P}=0.023)$, high levels of $\mathrm{CEA}(\mathrm{HR}=2.315,95 \% \mathrm{CI}$ : 
Table IV. Univariate and multivariate analysis of prognostic factors for overall survival.

\begin{tabular}{|c|c|c|c|c|}
\hline \multirow[b]{2}{*}{ Prognostic factor } & \multicolumn{2}{|l|}{ Univariate } & \multicolumn{2}{|c|}{ Multivariate } \\
\hline & $\mathrm{HR}(95 \% \mathrm{CI})$ & P-value & $\mathrm{HR}(95 \% \mathrm{CI})$ & P-value \\
\hline MUC1 ( $\geq 0.056$ vs. $<0.056$, ratio to GAPDH) & $2.233(1.214-4.485)$ & 0.011 & $2.364(1.127-4.960)$ & 0.023 \\
\hline CEA $(\geq 20 \mathrm{ng} / \mathrm{ml}$ vs. $<20 \mathrm{ng} / \mathrm{ml})$ & $2.197(1.106-4.365)$ & 0.025 & $2.315(1.081-4.958)$ & 0.031 \\
\hline CA-19-9 ( $\geq 37 \mathrm{ng} / \mathrm{ml}$ vs. $<37 \mathrm{ng} / \mathrm{ml})$ & $2.675(1.368-5.231)$ & 0.004 & $2.303(1.109-4.780)$ & 0.025 \\
\hline Differentiation (poor vs. well) & $2.284(1.102-4.736)$ & 0.026 & $1.102(0.485-2.502)$ & 0.816 \\
\hline Tumor diameter $(>5 \mathrm{~cm}$ vs. $\leq 5 \mathrm{~cm})$ & $2.236(1.110-4.503)$ & 0.024 & $1.461(1.127-4.960)$ & 0.320 \\
\hline LN metastasis (yes vs. no) & $4.440(1.916-10.288)$ & 0.001 & $2.846(1.259-6.436)$ & 0.012 \\
\hline T stage (T2-4 vs. T1) & $1.435(0.995-2.070)$ & 0.053 & & \\
\hline Surrounding tissue invasion (yes vs. no) & $2.612(0.797-8.557)$ & 0.113 & & \\
\hline Tumor number (solitary vs. multiple) & $1.603(0.838-3.064)$ & 0.154 & & \\
\hline Nerve invasion (yes vs. no) & $1.117(0.268-4.645)$ & 0.879 & & \\
\hline Vascular invasion (yes vs. no) & $0.798(0.378-1.681)$ & 0.552 & & \\
\hline Bile duct invasion (presence vs. absence) & $1.995(0.762-5.018)$ & 0.163 & & \\
\hline
\end{tabular}

LN, lymph node; HR, hazard ratio; CA, cancer antigen.

A

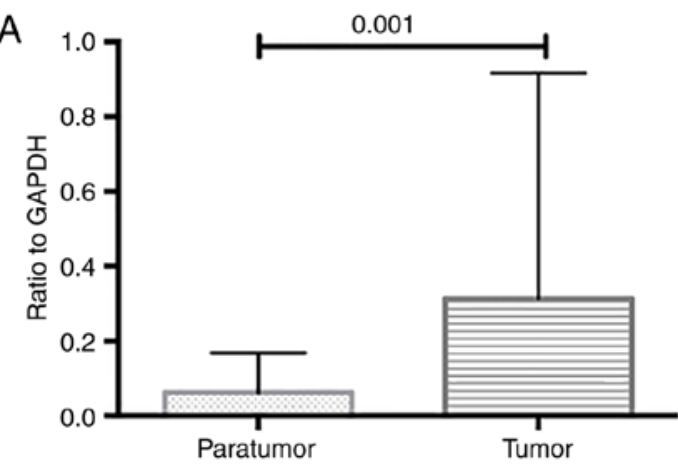

C

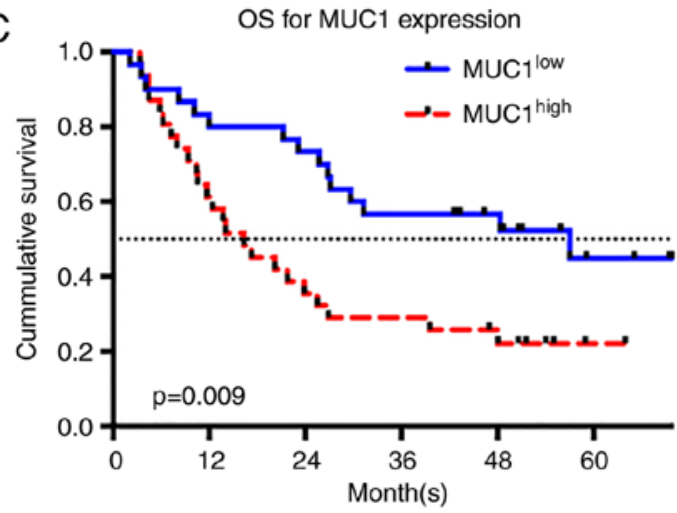

B

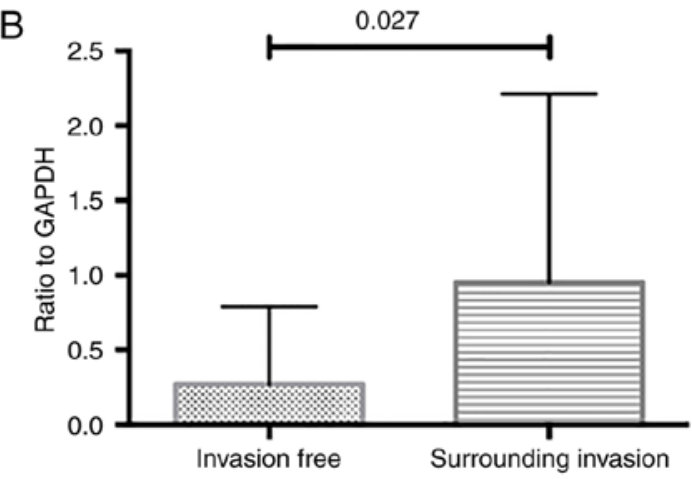

D

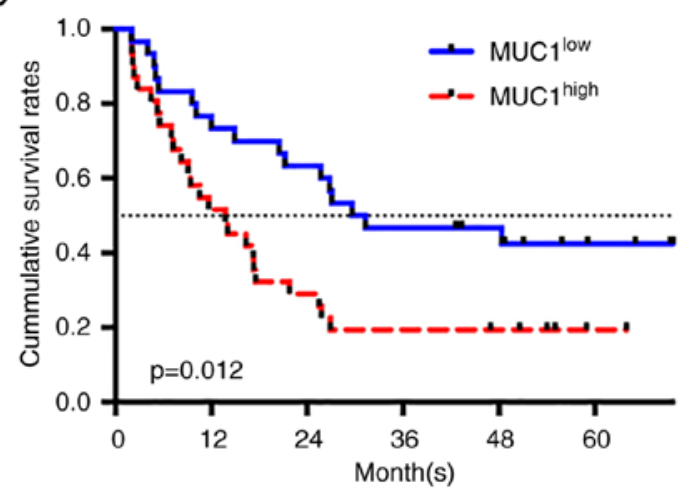

Figure 2. Clinical validation of MUC1 expression and its prognostic significance. (A) The levels of MUC1 were significantly greater in the ICC tumor tissues compared with the matched paratumor tissues. (B) MUC1 was also significantly higher in tumors with surrounding tissue invasion, compared with the tumors that did not invade. All data were presented as the mean + standard deviation. (C and D) MUC1 was a prognostic predictor for (C) OS and (D) RFS. MUC1 low refers to the group with low expression of MUC1 in ICC tissue and MUC1 ${ }^{\text {high }}$ to the group with high expression of MUC1 in ICC tissue. MUC1, mucin 1; ICC, intrahepatic cholangiocarcinoma; OS, overall survival; RFS, recurrence-free survival.

1.081-4.958, $\mathrm{P}=0.031)$ and $\mathrm{CA} 19-9(\mathrm{HR}=2.303,95 \% \mathrm{CI}$ : $1.109-4.780, \mathrm{P}=0.025)$, and the presence of lymph node metastasis $(\mathrm{HR}=2.846,95 \% \mathrm{CI}: 1.259-6.436, \mathrm{P}=0.012)$ were independent prognostic factors for OS (Table IV). A high level of MUC1 was also a prognostic factor for RFS (univariate analysis $\mathrm{HR}=2.187,95 \% \mathrm{CI}$ : $1.171-4.083, \mathrm{P}=0.014$; multivariate 
Table V. Univariate and multivariate analysis of prognostic factors for recurrence-free survival.

\begin{tabular}{|c|c|c|c|c|}
\hline \multirow[b]{2}{*}{ Prognostic factor } & \multicolumn{2}{|c|}{ Univariate } & \multicolumn{2}{|c|}{ Multivariate } \\
\hline & $\mathrm{HR}(95 \% \mathrm{CI})$ & P-value & $\mathrm{HR}(95 \% \mathrm{CI})$ & P-value \\
\hline MUC1 ( $\geq 0.056$ vs. $<0.056$, ratio to GAPDH) & $2.187(1.171-4.083)$ & 0.014 & $2.552(1.294-5.032)$ & 0.007 \\
\hline Bile duct invasion (yes vs. No) & $2.445(1.022-5.851)$ & 0.045 & $3.979(1.449-10.928)$ & 0.007 \\
\hline Tumor diameter $(>5 \mathrm{~cm}$ vs. $\leq 5 \mathrm{~cm})$ & $1.977(1.026-3.810)$ & 0.042 & $1.130(0.509-2.510)$ & 0.764 \\
\hline T stage (T2-4 vs. T1) & $1.468(1.039-2.073)$ & 0.029 & $1.403(0.931-2.115)$ & 0.106 \\
\hline LN metastasis (yes vs. no) & $2.449(1.256-4.776)$ & 0.009 & $2.053(1.042-4.046)$ & 0.038 \\
\hline Surrounding tissue invasion (yes vs. no) & $2.045(0.628-6.656)$ & 0.235 & & \\
\hline $\mathrm{CEA}(\geq 20 \mathrm{ng} / \mathrm{ml}$ vs. $<20 \mathrm{ng} / \mathrm{ml})$ & $1.108(10.567-2.167)$ & 0.764 & & \\
\hline CA-19-9 ( $\geq 37 \mathrm{ng} / \mathrm{ml}$ vs. $<37 \mathrm{ng} / \mathrm{ml})$ & $0.828(0.452-1.516)$ & 0.540 & & \\
\hline Differentiation (poor vs. well) & $1.930(0.940-3.960$ & 0.073 & & \\
\hline Tumor number (solitary vs. multiple) & $1.504(0.798-2.836)$ & 0.207 & & \\
\hline Nerve invasion (yes vs. no) & $2.217(0.679-7.239)$ & 0.187 & & \\
\hline Vascular invasion (yes vs. no) & $0.971(0.488-1.933)$ & 0.933 & & \\
\hline
\end{tabular}

LN, lymph node; HR, hazard ratio; CA, cancer antigen.
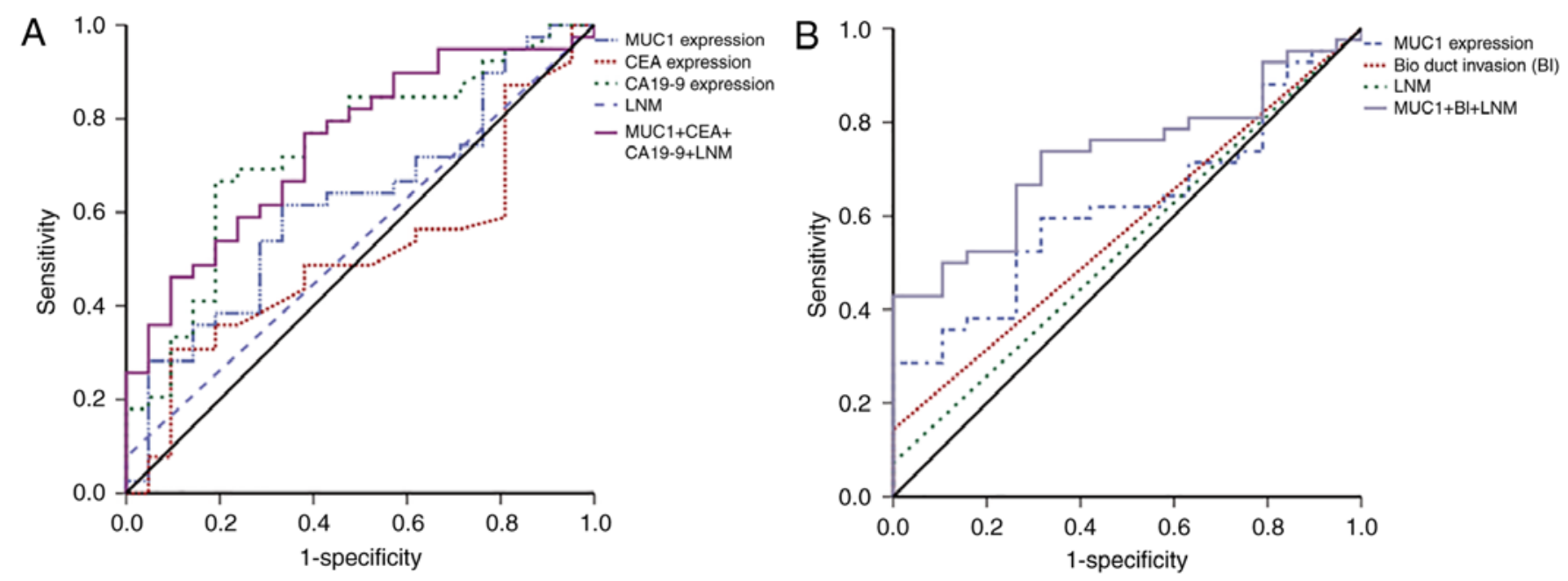

Figure 3. ROC curves for OS and RFS. (A) ROC curve for OS. (B) ROC curve for RFS. LNM, lymph node metastasis; BI, bile duct invasion; CA19-9, cancer antigen 19-9; ROC, receiver operating characteristic; MUC1, mucin 1; OS, overall survival; RFS, recurrence-free survival.

analysis $\mathrm{HR}=2.552$, 95\%CI: $1.294-5.032, \mathrm{P}=0.007)$. Other independent risk factors for RFS time were the presence of lymph node metastasis (HR=2.053 95\%CI: 1.042-4.046, 95\% CI, $\mathrm{P}=0.038)$ and bile duct invasion $(\mathrm{HR}=3.979$ 95\% CI: $1.449-10.928, \mathrm{P}=0.007$; Table V).

ROC curve analysis of OS and RFS was then used to examine the predictive precision of these independent prognostic factors. MUC1 expression levels alone was not a strong predictor of OS (AUC $=0.623$ ), but the combination of the serum levels of MUC1, CEA and CA19-9, and lymph node metastasis, was a better predictor of OS $(\mathrm{AUC}=0.746$; Fig. 3A and Table VI). The ROC curve for RFS indicated that MUC1 combined with bile duct invasion and lymph node metastasis was the best predictor of RFS (AUC $=0.729$; Fig. 3B and Table VI).

\section{Discussion}

ICC has a low incidence but is associated with high mortality. Due to the lack of effective treatments, the incidence of ICC-associated mortality is increasing worldwide $(10,27,28)$. Gene dysregulation contributes to the genesis of ICC (10). Most of the gene expression studies that have examined how dysregulated genes contribute to the pathogenesis of ICC did not include sufficient samples to identify relevant genes or had no clinical data for validation (23-26). Therefore, 
Table VI. Receiver operating characteristic analysis for OS and RFS.

\begin{tabular}{lc}
\hline A, OS & \\
\hline Parameter & AUC (95\%CI) \\
\hline MUC1 & $0.623(0.476-0.769)$ \\
CEA & $0.509(0.357-0.660)$ \\
CA19-9 & $0.734(0.600-0.867)$ \\
LNM & $0.538(0.388-0.689)$ \\
Combined Co-index 1 & $0.746(0.620-0.872)$ \\
\hline
\end{tabular}

\section{$\mathrm{B}, \mathrm{RFS}$}

\begin{tabular}{lc}
\hline Parameter & AUC (95\%CI) \\
\hline MUC1 & $0.622(0.480-0.763)$ \\
BI & $0.571(0.423-0.720)$ \\
LNM & $0.536(0.382-0.689)$ \\
Combined Co-index 2 & $0.729(0.605-0.854)$ \\
\hline
\end{tabular}

The status of OS and RFS used to calculate the AUC after the very last follow-up appointment (2016.06.30). LNM refers to the presence of lymph node metastasis; BI refers to the presence of bile duct invasion. Combined co-index 1, MUC1 + CEA+ CA19-9 + LNM; Combined Co-index 2, MUC1 + BI + LNM. MUC1, mucin 1; OS, overall survival; RFS, recurrence-free survival; $\mathrm{CA}$, cancer antigen.

a combined approach that included the use of integrated Bioinformatics analysis and clinical validation was used in the present study to identify genes involved in ICC. Lack of availability of raw expression datasets and difficulty in achieving rigorous normalization and integration over platforms are obstacles for the meta-analysis of gene expression data. In the present study, the RRA method was used to overcome these disadvantages (16).

MUC1 is a heterodimeric type I transmembrane glycoprotein expressed on the surface of the epithelium of most organs (e.g., mammary, gastric, respiratory, urinary, and reproductive tract) $(29,30)$. MUC1 interacts with $\mathrm{p} 53$, which results in the inhibition of p53-mediated apoptosis (31). It also interacts with $\beta$-catenin to initiate the epithelial-mesenchymal transition that promotes the onset of metastasis (32). Overexpression of MUC1 is associated with the malignancy of ICC and indicates a poor outcome $(33,34)$. However, most of the above-mentioned studies were single-center and single-ethnicity investigations. In the present study, an integrated Bioinformatics analysis of data from multiple centers (241 samples) and multiple ethnicities was performed. The results suggested that MUC1 regulates ICC tumor invasion. Certain studies have indicated that $\mathrm{MUC1}$ expression is a predictor for OS in ICC patients $(34,35)$, but few have detected a correlation between MUC1 expression and RFS. All patients included in the combined cohort of the present study had comprehensive clinical information and the longest follow-up was $>5$ years. Although the cohort of patients was heterogenous with $\mathrm{T} 1$ or $\mathrm{T} 2$ tumors accounting for $\sim 50 \%$, there was no significant difference in $\mathrm{T}$ stage between the MUC1 $1^{\text {high }}$ group and the $\mathrm{MUC}^{\text {low }}$ group $(\mathrm{P}=0.18)$. Most patients with T3 or T4 tumors decided not to receive any surgery and accordingly, no samples were collected. Furthermore, patients with T3 and T4 tumors usually received pre-operative treatment and were ruled out based on the inclusion criteria. These circumstances led to heterogeneity. The present results revealed that the expression level of MUC1 was an independent prognostic factor for OS and RFS. The ROC analysis revealed that a model combining MUC1 expression, bile duct invasion and lymph node metastasis may be used to predict RFS (AUC=0.729). Using MUC1 as a predictor for RFS and OS may help identify patients with a high risk of recurrence, which may aid in the selection and development of post-operative treatment and monitoring protocols.

In the present study, a novel approach that may be used to study ICC and improve clinical practice was developed. To the best of our knowledge, the present study was the first to combine integrated Bioinformatics analysis with clinical validation to identify biomarkers for ICC. Use of the GEO and TCGA datasets provided sufficient evidence to prove the association of MUC1 with ICC. The large number of clinical samples included increases the validity of the present results. ICC is a cancer type with high malignancy, which lacks effective treatments $(10,27,28)$. MUC1 has been previously reported to be a potential target for anti-cancer therapies $(12,36,37)$. A glycosylated tripartite vaccine that targets MUC1 is under development (38). Another MUC1 vaccine (ONT-10) has been tested in a phase I clinical trial (39). The results of the present study provided further insight that will be useful for the development of comprehensive treatments for ICC. However, studies that use larger sample sizes should be performed to validate the present results. In vitro and in vivo experiments are also required to determine the potential mechanisms by which MUC1 drives tumorigenesis.

In the present study, it was identified that MUC1 was upregulated in ICC tissues and that it was associated with prognosis. Clinical validation revealed that a co-index including MUC1 and other clinical parameters predicts recurrence of ICC with high accuracy, which may support the selection and development of post-operative treatment and monitoring protocols.

\section{Acknowledgements}

The results shown here are in whole or part based upon data generated by the TCGA Research Network: https://www. cancer.gov/tcga.

\section{Funding}

The present study was supported by the National Natural Science Foundation of China (grant nos. 81401929, 81572823 and 2017ZX10203204), the Shanghai Rising-Star Program (grant no. 16QA1401000), the Shanghai Hospital Development Center (grant no. SHDC12015104) and the National Key Research and Development Program (grant no. 2016YFC0902400). 


\section{Availability of data and materials}

The datasets generated and/or analysed during the current study are available in the GEO: GSE89749 (https:/www.ncbi.nlm. nih.gov/geo/query/acc.cgi?acc=GSE89749), GSE76297 (https:// www.ncbi.nlm.nih.gov/geo/query/acc.cgi?acc=GSE76297), GSE57555 (https://www.ncbi.nlm.nih.gov/geo/query/ acc.cgi?acc=GSE57555), GSE32879 (https://www.ncbi. nlm.nih.gov/geo/query/acc.cgi?acc=GSE32879); and GSE26566 (https://www.ncbi.nlm.nih.gov/geo/query/acc. cgi?acc=GSE26566) and TCGA (https://cancergenome.nih. gov/). All other data are included in this published article and its supplementary data.

\section{Authors' contributions}

FYC designed the study, analysed the data and drafted the manuscript. $\mathrm{CZ}, \mathrm{XYZ}$ and $\mathrm{KQZ}$ collected and verified the data. YFP and LY analyzed and interpreted the data. JF, JZ, JH and $\mathrm{ZW}$ designed the study, interpreted the data, revised the manuscript and made the decision to submit it for publication. All authors read and approved the final manuscript.

\section{Ethics approval and consent to participate}

The present study was approved by the Ethics Committee of Zhongshan Hospital, Fudan University (Shanghai, China). Written informed consent was obtained from each patient.

\section{Patient consent for publication}

Not applicable.

\section{Competing interests}

The authors declare that they have no competing interests.

\section{References}

1. Aljiffry M, Abdulelah A, Walsh M, Peltekian K, Alwayn I and Molinari M: Evidence-based approach to cholangiocarcinoma: A systematic review of the current literature. J Am Coll Surg 208: 134-147, 2009

2. Buettner S, van Vugt JL, IJzermans JN and Groot Koerkamp B: Intrahepatic cholangiocarcinoma: Current perspectives. Onco Targets Ther 10: 1131-1142, 2017.

3. Schlinkert RT, Nagorney DM, Van Heerden JA and Adson MA: Intrahepatic cholangiocarcinoma: Clinical aspects, pathology and treatment. HPB Surg 5: 95-102, 1992.

4. Endo I, Gonen M, Yopp AC, Dalal KM, Zhou Q, Klimstra D, D'Angelica M, DeMatteo RP, Fong Y, Schwartz L, et al: Intrahepatic cholangiocarcinoma: Rising frequency, improved survival, and determinants of outcome after resection. Ann Surg 248: 84-96, 2008.

5. Dodson RM, Weiss MJ, Cosgrove D, Herman JM, Kamel I, Anders R, Geschwind JF and Pawlik TM: Intrahepatic cholangiocarcinoma: Management options and emerging therapies. J Am Coll Surg 217: 736-750.e4, 2013.

6. Shaib YH, Davila JA, McGlynn K and El-Serag HB: Rising incidence of intrahepatic cholangiocarcinoma in the United States: A true increase? J Hepatol 40: 472-477, 2004.

7. Yamashita Y, Taketomi A, Morita K, Fukuhara T, Ueda S, Sanefuji K, Iguchi T, Kayashima H, Sugimachi K and Maehara Y: The impact of surgical treatment and poor prognostic factors for patients with intrahepatic cholangiocarcinoma: Retrospective analysis of 60 patients. Anticancer Res 28: 2353-2359, 2008.
8. Weber SM, Ribero D, O'Reilly EM, Kokudo N, Miyazaki M and Pawlik TM: Intrahepatic cholangiocarcinoma: Expert consensus statement. HPB (Oxford) 17: 669-680, 2015.

9. Popat K, McQueen K and Feeley TW: The global burden of cancer. Best Pract Res Clin Anaesthesiol 27: 399-408, 2013.

10. Bridgewater J, Galle PR, Khan SA, Llovet JM, Park JW, Patel T, Pawlik TM and Gores GJ: Guidelines for the diagnosis and management of intrahepatic cholangiocarcinoma. J Hepatol 60: 1268-1289, 2014.

11. Rao CV, Asch AS and Yamada HY: Frequently mutated genes/pathways and genomic instability as prevention targets in liver cancer. Carcinogenesis 38: 2-11, 2017.

12. Rizvi S and Gores GJ: Emerging molecular therapeutic targets for cholangiocarcinoma. J Hepatol 67: 632-644, 2017.

13. Rahnemai-Azar AA, Weisbrod A, Dillhoff M, Schmidt C and Pawlik TM: Intrahepatic cholangiocarcinoma: Molecular markers for diagnosis and prognosis. Surg Oncol 26: 125-137, 2017.

14. Wirapati P, Sotiriou C, Kunkel S, Farmer P, Pradervand S, Haibe-Kains B, Desmedt C, Ignatiadis M, Sengstag T, Schütz F, et al: Meta-analysis of gene expression profiles in breast cancer: Toward a unified understanding of breast cancer subtyping and prognosis signatures. Breast Cancer Res 10: R65, 2008.

15. Võsa U,Kolde R, Vilo J, Metspalu A and Annilo T: Comprehensive meta-analysis of microRNA expression using a robust rank aggregation approach. Methods Mol Biol 1182: 361-373, 2014.

16. Kolde R, Laur S, Adler P and Vilo J: Robust rank aggregation for gene list integration and meta-analysis. Bioinformatics 28 : 573-580, 2012.

17. Edgar R, Domrachev M and Lash AE: Gene expression omnibus: NCBI gene expression and hybridization array data repository. Nucleic Acids Res 30: 207-210, 2002.

18. Smyth GK: In Bioinformatics and Computational Biology Solutions Using R and Bioconductor (Springer-Verlag, New York), Statistics for Biology and Health, pp397-420, 2005.

19. McCarthy DJ, Chen Y and Smyth GK: Differential expression analysis of multifactor RNA-Seq experiments with respect to biological variation. Nucleic Acids Res 40: 4288-4297, 2012.

20. Livak KJ and Schmittgen TD: Analysis of relative gene expression data using real-time quantitative PCR and the 2(-Delta Delta C(T)) method. Methods 25: 402-408, 2001.

21. Amin MB: AJCC Cancer Staging Manual, 8th edition

22. Jusakul A, Cutcutache I, Yong CH, Lim JQ, Huang MN, Padmanabhan N, Nellore V, Kongpetch S, Ng AWT, Ng LM, et al: Whole-Genome and epigenomic landscapes of etiologically distinct subtypes of cholangiocarcinoma. Cancer Discov 7: 1116-1135, 2017.

23. Chaisaingmongkol J, Budhu A, Dang H, Rabibhadana S, Pupacdi B, Kwon SM, Forgues M, Pomyen Y, Bhudhisawasdi V, Lertprasertsuke $\mathrm{N}$, et al: Common molecular subtypes among Asian hepatocellular carcinoma and cholangiocarcinoma. Cancer Cell 32: 57-70.e3, 2017.

24. Murakami Y, Kubo S, Tamori A, Itami S, Kawamura E, Iwaisako K, Ikeda K, Kawada N, Ochiya T and Taguchi YH: Comprehensive analysis of transcriptome and metabolome analysis in Intrahepatic Cholangiocarcinoma and Hepatocellular Carcinoma. Sci Rep 5: 16294, 2015.

25. Oishi N, Kumar MR, Roessler S, Ji J, Forgues M, Budhu A, Zhao X, Andersen JB, Ye QH, Jia HL, et al: Transcriptomic profiling reveals hepatic stem-like gene signatures and interplay of miR-200c and epithelial-mesenchymal transition in intrahepatic cholangiocarcinoma. Hepatology 56: 1792-1803, 2012.

26. Andersen JB, Spee B, Blechacz BR, Avital I, Komuta M, Barbour A, Conner EA, Gillen MC, Roskams T, Roberts LR, et al: Genomic and genetic characterization of cholangiocarcinoma identifies therapeutic targets for tyrosine kinase inhibitors. Gastroenterology 142: 1021-1031.e15, 2012.

27. Guro H, Kim JW, Choi Y, Cho JY, Yoon YS and Han HS: Multidisciplinary management of intrahepatic cholangiocarcinoma: Current approaches. Surg Oncol 26: 146-152, 2017.

28. Kaczynski J, Hansson G and Wallerstedt S: Incidence, etiologic aspects and clinicopathologic features in intrahepatic cholangiocellular carcinoma-a study of 51 cases from a low-endemicity area. Acta Oncol 37: 77-83, 1998.

29. Brayman M, Thathiah A and Carson DD: MUC1: A multifunctional cell surface component of reproductive tissue epithelia. Reprod Biol Endocrinol 2: 4, 2004.

30. Aplin JD, Hey NA and Li TC: MUC1 as a cell surface and secretory component of endometrial epithelium: Reduced levels in recurrent miscarriage. Am J Reprod Immunol 35: 261-266, 1996. 
31. Wei X, Xu H and Kufe D: Human MUC1 oncoprotein regulates p53-responsive gene transcription in the genotoxic stress response. Cancer Cell 7: 167-178, 2005.

32. Roy LD, Sahraei M, Subramani DB, Besmer D, Nath S, Tinder TL, Bajaj E, Shanmugam K, Lee YY, Hwang SI, et al: MUC1 enhances invasiveness of pancreatic cancer cells by inducing epithelial to mesenchymal transition. Oncogene 30: 1449-1459, 2011

33. Higashi M, Yonezawa S, Ho JJ, Tanaka S, Irimura T, Kim YS and Sato E: Expression of MUC1 and MUC2 mucin antigens in intrahepatic bile duct tumors: Its relationship with a new morphological classification of cholangiocarcinoma. Hepatology 30 : 1347-1355, 1999.

34. Park SY, Roh SJ, Kim YN, Kim SZ, Park HS, Jang KY Chung MJ, Kang MJ, Lee DG and Moon WS: Expression of MUC1, MUC2, MUC5AC and MUC6 in cholangiocarcinoma: Prognostic impact. Oncol Rep 22: 649-657, 2009.

35. Ruys AT, Groot Koerkamp B, Wiggers JK, Klümpen HJ, ten Kate FJ and van Gulik TM: Prognostic biomarkers in patients with resected cholangiocarcinoma: A systematic review and meta-analysis. Ann Surg Oncol 21: 487-500, 2014.
36. Pillai K, Pourgholami MH, Chua TC and Morris DL: MUC1 as a potential target in anticancer therapies. Am J Clin Oncol 38: 108-118, 2015.

37. Sasaki M, Nakanuma Y and Kim YS: Characterization of apomucin expression in intrahepatic cholangiocarcinomas and their precursor lesions: An immunohistochemical study. Hepatology 24: 1074-1078, 1996.

38. Lakshminarayanan V, Thompson P, Wolfert MA, Buskas T, Bradley JM, Pathangey LB, Madsen CS, Cohen PA, Gendler SJ and Boons GJ: Immune recognition of tumor-associated mucin MUC1 is achieved by a fully synthetic aberrantly glycosylated MUC1 tripartite vaccine. Proc Natl Acad Sci USA 109: 261-266, 2012.

39. Nemunaitis J, Bedell C, Klucher K, Vo A and Whiting S: Phase 1 dose escalation of ONT-10, a therapeutic MUC1 vaccine, in patients with advanced cancer. J Immunother Cancer 1 (Suppl 1): P240, 2013.

This work is licensed under a Creative Commons Attribution-NonCommercial-NoDerivatives 4.0 International (CC BY-NC-ND 4.0) License. 